\title{
Selection of Modulating Functions' Design Parameters for Estimation Problems
}

\author{
Sharefa Asiri ${ }^{1,3}$, Da-Yan Liu ${ }^{2}$, and Taous-Meriem Laleg-Kirati ${ }^{3}$
}

\begin{abstract}
In this paper, an effective algorithm for selecting the design parameters in the modulating functions-based method (MFBM) is introduced. The appropriate selection of these parameters improves the performance of the estimation approach and reduces the computational cost. The effectiveness and robustness of the algorithm are shown through numerical simulations in a noisy environment.
\end{abstract}

Index Terms-Modulating function, Parameters selection, KdV equation

\section{INTRODUCTION}

$\mathbf{P}$ Arameters of differential equation (DE) models are often unknown and are usually estimated from available measurements. However, measurements are usually noisy in practice. Hence, differentiating them is a well-known illposed problem. In other words, differentiating measurements that are corrupted with small errors may lead to a large error in the solution. In addition, some estimation methods, such as optimization-based methods, require solving the DE system. However, analytic solutions of some DEs are not always easy to compute. Thus, solutions of these equations are usually approximated numerically, this increases the difficulty of solving estimation problems in DEs, especially in the case of higher-order nonlinear DEs.

Modulating functions-based method (MFBM) is among the recent active and effective methods for real-time estimation of input, states, and parameters of systems governed by DEs [1] [6]. The MFBM consists of a kernel-based linear integral operator in which the kernel is given by certain functions called modulating functions. Then by using integration by parts, the derivatives of the measurements are transferred to the ones of the modulating functions, which are known analytically. Hence, numerical instabilities coming from differentiating the noisy measurements are avoided. Moreover, the estimation problem can be reformulated as the resolution of an algebraic system of equations and hence can avoid solving the DE. In addition, the MFBM provides a non-asymptotic solution of the estimation problem with excellent robustness properties, hence is interesting in many real processes. Further, the MFBM is independent of the initial (and boundary if any) conditions which are unknowns in many applications. The features of the MFBM are similar to those in the algebraic estimation methods

\footnotetext{
${ }^{1}$ Department of Mathematics, King Abdulaziz University, Jeddah 2158980200, Saudi Arabia smaasiri@kau.edu.sa

${ }^{2}$ INSA Centre Val de Loire, Université d'Orléans, PRISME EA 4229, Bourges Cedex 18022, France dayan.liueinsa-cvl.fr

${ }^{3}$ Computer, Electrical and Mathematical Science and Engineering Division, King Abdullah University of Science and Technology (KAUST), Thuwal 23955-6900, Saudi Arabia taousmeriem. laleg@kaust.edu.sa
}

[7]. In addition, the MFBM can be considered as a generalization of the algebraic estimation method in some cases (see, e.g., [8]). Very recently, an intelligent proportional-integralderivative controller has been combined with the MFBM to enhance the performance and the robustness of the controlled system [9]. For more details about the MFBM, the reader can refer to [10] and [11].

The analyze on the selection of modulating functions is important to improve the estimation results, for example, the study of choosing design parameters. However, tuning the design parameters is not a trivial and systematic task as it depends on the considered estimation problem, on the noise corrupting the measurements, and on many other factors such as the number of grids used in the discrete case. The works in [12][14] provide a way for selecting these parameters in signal estimation problems. For parameters estimation problems, the authors in [15] described the statistical properties of the modulating functions solution, and then used them to provide a guideline for choosing the number of modulating functions, which is one of the MFBM design parameters.

Compared to [15], the contributions of this paper can be summarized as follows.

- A sensitivity analysis of the modulating functions' position and width is studied.

- Based on that, an effective algorithm for selecting all the design parameters of the modulating functions, i.e. constructing the modulating functions, is provided. Consequently, the performance of the method is enhanced.

- A strategy for selecting the minimum number (which is equal to the number of unknowns) of appropriate modulating functions is proposed. Hence, the computational load is reduced by reducing the size of the constructed algebraic system to a square one. As a result, the solution to the estimation problem is provided by the solution of the reduced square one.

The effectiveness of the algorithm is illustrated on a high-order nonlinear partial differential equation which is the fifth-order Korteweg-de Vries (fKdV) equation. The algorithm presented in this paper can be used offline but an extension to real-time estimation will be investigated in future work.

\section{PRELIMINARIES}

Throughout this work, the symbols $\mathbb{N}^{*}, \mathbb{R}$, and $\mathbb{R}^{*}$ denote the sets of positive integers, real numbers, and non-zero real numbers, respectively. The definition and some properties of modulating functions are presented in this section. 
Definition 1. [16] A function $\phi(x) \neq 0$ is called a modulating function of order $l\left(l \in \mathbb{N}^{*}\right)$ on $[0, L]$ if it satisfies:

$$
\left\{\begin{array}{l}
\phi(x) \in C^{l}([0, L]), \\
\phi^{(p)}(0)=\phi^{(p)}(L)=0, \quad \text { for } p=0,1, \ldots, l-1
\end{array}\right.
$$

where $L>0$ and $(p)$ refers to the derivative order.

Lemma 1. Let $\mathbb{P}^{\bar{n}}$ be a differential operator defined on $C^{\bar{n}}([0, L])$ such that $\mathbb{P}^{\bar{n}} u(x)=\sum_{s=1}^{\bar{n}} b_{s}(x) \frac{\mathrm{d}^{s}}{\mathrm{~d} x^{s}} u(x)$, where $x \in[0, L], u \in C^{\bar{n}}([0, L])$, and $b_{s}(x)$, for $s=1, \ldots, \bar{n}$, are sufficiently smooth coefficients. If $\phi(x)$ is a modulating function of order $l(l \geq \bar{n})$ on $[0, L]$, then

$$
\int_{0}^{L} \mathbb{P}^{\bar{n}} u(x) \phi(x) \mathrm{d} x=\int_{0}^{L} u(x) \mathbb{Q}^{\bar{n}} \phi(x) \mathrm{d} x,
$$

where $\mathbb{Q}^{\bar{n}} \phi(x)=\sum_{s=1}^{\bar{n}}(-1)^{s} \frac{\mathrm{d}^{s}}{\mathrm{~d} x^{s}}\left[b_{s}(x) \phi(x)\right]$.

Proof. The application of the integration by parts formula to (2) leads to the following equation:

$$
\begin{array}{r}
\int_{0}^{L} \mathbb{P}^{\bar{n}} u(x) \phi(x) \mathrm{d} x=\left.\sum_{s=1}^{\bar{n}} \phi(x) b_{s}(x) \frac{\mathrm{d}^{(s-1)}}{\mathrm{d} x^{(s-1)}} u(x)\right|_{x=0} ^{x=L} \\
-\sum_{s=1}^{\bar{n}} \int_{0}^{L} \frac{\mathrm{d}^{(s-1)}}{\mathrm{d} x^{(s-1)}} u(x) \frac{\mathrm{d}}{\mathrm{d} x}\left[b_{s}(x) \phi(x)\right] \mathrm{d} x .
\end{array}
$$

In the Right Hand Side (R.H.S) of (3), the first term equals to zero from (1b). Using successive integration by parts and using the property in (1b) leads to the R.H.S of (2).

The application of Lemma 1 will lead to an important advantage of using modulating functions which is the transfer of the derivatives from $u$ to modulating functions. This reduces the instability of the estimation results as $u$ is usually unknown and noisy. Moreover, the integral operators used in the MFBM ensure good robustness properties of the method.

\section{Coefficients Estimation Using the Modulating FUnCTIONS-BASED METHOD}

\section{A. Problem Formulation}

Consider the following form for one-dimensional partial differential equations defined on $\Omega:=(0, L) \times(0, T]$ :

$$
\mathbb{T} u(x, t)+\mathbb{P} u(x, t)=f(x, t),
$$

with appropriate initial and boundary conditions, where $x$ is the space variable, $t$ is the time variable, and $f(x, t)$ is the source term. $\mathbb{T}$ and $\mathbb{P}$ are temporal and spatial partial differential operators such that:

$$
\mathbb{T} u(x, t)=\sum_{r=0}^{\bar{m}} c_{r} \frac{\partial^{r} u(x, t)}{\partial t^{r}},
$$

and

$$
\mathbb{P} u(x, t)=\sum_{s=0}^{\bar{n}} \sum_{j=1}^{p} b_{s, j} \frac{\partial^{s} u^{j}(x, t)}{\partial x^{s}},
$$

where $c_{r} \in \mathbb{R}$ for $r=0, \ldots, \bar{m}$, and $b_{s, j}$ for $s=0, \ldots, \bar{n}$, $j=1, \ldots, p$, are constant coefficients. The estimation problem under investigation in this paper can be formulated as follows:

EP: Given $\left\{\left.c_{r} \frac{\partial^{r} u(x, t)}{\partial t^{r}}\right|_{t=t^{*}}\right\}_{r=0}^{\bar{m}}$ at a fixed time $t^{*}$, find $b_{s, j}$, for $s=0, \ldots, \bar{n}, j=1, \ldots, p$.

\section{B. Estimation Procedure}

The MFBM solution to the estimation problem EP is given in the following proposition.

Proposition 1. Let $b_{s, j}$, for $s=0, \ldots, \bar{n}, j=1, \ldots, p$, be unknown coefficients in (4), and let $\left\{\phi_{m}(x)\right\}_{m=1}^{m=M}$ be a class of linearly independent modulating functions of order at least $\bar{n}$ such that $M \geq p(\bar{n}+1)$. Then, the unknown coefficients can be obtained by solving the linear system:

$$
\mathcal{A} \Gamma=K,
$$

where $\mathcal{A}=\left[A_{0}, A_{1}, \ldots, A_{\bar{n}}\right] \in \mathbb{R}^{M \times p(\bar{n}+1)}$ such that the entries of the matrix $A_{s}$ and the vector $K$ are given by: for $s=0, \ldots, \bar{n}, j=1, \ldots, p, m=1, \ldots, M$,

$$
\begin{aligned}
& \left(a_{s}\right)_{m j}=(-1)^{s} \int_{0}^{L} u^{j}\left(x, t^{*}\right) \frac{d^{s} \phi_{m}(x)}{d x^{s}} \mathrm{~d} x, \\
& k_{m}=\int_{0}^{L}\left[f\left(x, t^{*}\right)-\mathbb{T} u\left(x, t^{*}\right)\right] \phi_{m}(x) \mathrm{d} x,
\end{aligned}
$$

and $\Gamma$ is the vector of unknown coefficients.

Proof. First, multiplying (4), at fixed time $t^{*}$, by the modulating functions $\phi_{m}(x)$, for $m=1,2, \ldots, M$, and integrating over the space interval lead to:

$$
\begin{aligned}
\int_{0}^{L} \mathbb{T} u\left(x, t^{*}\right) \phi_{m}(x) \mathrm{d} x & +\int_{0}^{L} \mathbb{P} u\left(x, t^{*}\right) \phi_{m}(x) \mathrm{d} x \\
& =\int_{0}^{L} f\left(x, t^{*}\right) \phi_{m}(x) \mathrm{d} x .
\end{aligned}
$$

Then, applying Lemma 1 to the second term in (10) gives:

$$
\begin{aligned}
& \sum_{s=0}^{\bar{n}} \sum_{j=1}^{p}(-1)^{s} b_{s, j} \int_{0}^{L} u^{j}\left(x, t^{*}\right) \frac{d^{s} \phi_{m}(x)}{d x^{s}} \mathrm{~d} x \\
= & \int_{0}^{L} f\left(x, t^{*}\right) \phi_{m}(x) \mathrm{d} x-\int_{0}^{L} \mathbb{T} u\left(x, t^{*}\right) \phi_{m}(x) \mathrm{d} x .
\end{aligned}
$$

The L.H.S of (11) represents the multiplication of the $m^{t h}$ row of $\mathcal{A}$ and the unknown vector $\Gamma$ while the R.H.S is the $m^{t h}$ entry of the vector $K$.

The solvability of (7) and the well-posedness of the MFBM solution have been discussed in [11] and [17].

The lower bound of the number of the modulating functions $M$ is equal to the number of unknowns. However, it has been shown in [11] that $M$ needs to be increased in some problems to give the method more robustness against corrupting noises. The next section investigates why using more modulating functions enhances the robustness feature.

\section{Modulating Functions' Parameters Selection STRATEGY}

\section{A. Preliminary tests}

We investigate here how a function and its derivatives modulate the measurement $u\left(x, t^{*}\right)$. More precisely, we examine the signals that come from the products of the modulating functions and their derivatives with the measurements, as these products are involved in the system matrices; and study their 
behavior with respect to $\phi_{1}, \phi_{2}, \ldots, \phi_{M}$. To this end, a set of synthetic data is generated using the following values: $L=60$, $T=50$, and $N_{x}=N_{t}=6001$, where $N_{x}$ and $N_{t}$ are space and time grid sizes, respectively.

Normalized power modulating functions are used in this paper, which have the following form:

$$
\phi(x)=\frac{\bar{\phi}(x)}{\|\bar{\phi}(x)\|_{L^{2}}},
$$

with

$$
\|\bar{\phi}(x)\|_{L^{2}}=\left(\int_{0}^{L}|\bar{\phi}(x)|^{2} \mathrm{~d} x\right)^{\frac{1}{2}}
$$

and

$$
\bar{\phi}(x)=(L-x)^{q_{1}} x^{q_{2}},
$$

where the exponents $q_{1}, q_{2} \in \mathbb{R}^{+}$are degrees of freedom (design parameters) which tune the order of the modulating functions. The order of the modulating function in (12) is $l=$ $\min \left\{\left\lfloor q_{1}\right\rfloor,\left\lfloor q_{2}\right\rfloor\right\}$. For the modulating functions, 56 functions have been generated from (12) as follows:

$$
\phi_{m}(x)=\frac{\bar{\phi}_{m}(x)}{\left\|\bar{\phi}_{m}(x)\right\|_{L^{2}}},
$$

with

$$
\bar{\phi}_{m}(x)=(L-x)^{13+m} x^{70-m},
$$

for $m=1, \ldots, 56$. To clarify the idea, let the measurement be, e.g., a signal that is almost zero outside a finite interval, as shown in the top-sub-figure in Fig. 1. The other sub-figures in Fig. 1 depict: first column, the modulating functions $\phi_{m}$ for different values of $m$; second column: the signals come from the product between $\phi_{m}$ and the measurement $u\left(x, t^{*}\right)$. The following observations on Fig. 1 are noteworthy:

- The important information in the measurement $u\left(x, t^{*}\right)$ is localized in the first third of the space interval.

- The non-zero part of the first modulating function $\phi_{1}(x)$ is approximately at the last third of the space interval, and then it starts moving to the left as $m$ increases.

- When the non-zero part of the modulating function is in a position totally different from the one of the measurement, then the product is a highly noisy signal which does not contain useful information, see, e.g., the plot of $\phi_{30} u\left(x, t^{*}\right)$ in Fig. 1 . Hence, it will negatively affect the estimation.

- When the non-zero parts of the modulating function and the measurement are relatively in the same interval, then the product is an informative signal that has almost zero noise outside the interval, see, e.g., the plot of $\phi_{50} u\left(x, t^{*}\right)$ in Fig. 1. Thus, these well-positioned modulating functions will certainly improve the effectiveness of the method.

From the above observations, we understand why usually using the minimum number of modulating functions in estimation problems did not provide good estimation results, and hence increasing $M$ was necessary. In other words, increasing the number of modulating functions usually increases the number of modulating functions that have well-localized non-zero parts and hence increases the robustness with respect to noises.
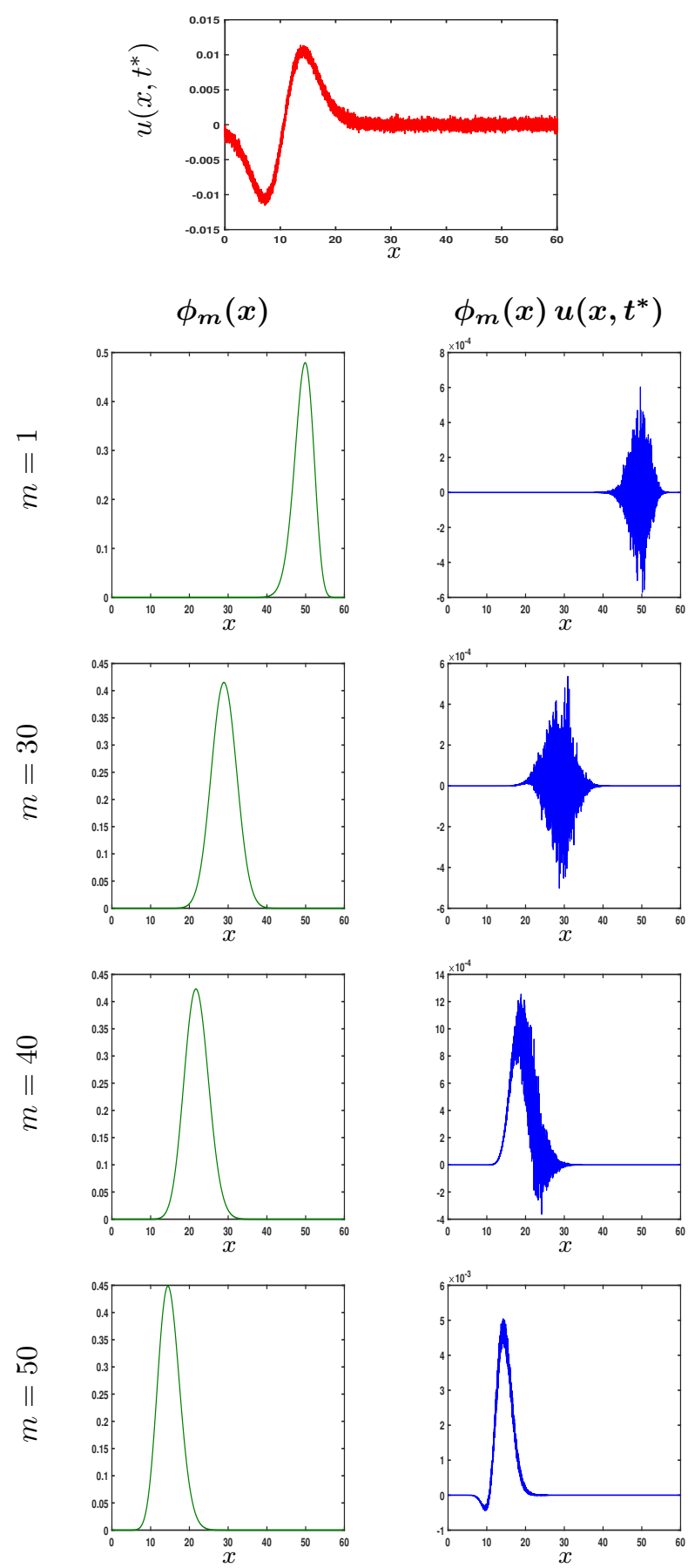

Fig. 1: Top figure: a noisy measurement $u\left(x, t^{*}\right)$ (Noise Level $=10 \%$ ). Bottom figure: the first column shows the modulating functions $\phi_{m}, m=1,30,40,50$; the second column represents the products between $\phi_{m}$ and the measurement $u\left(x, t^{*}\right)$ (the top figure).

The localization of the non-zero parts of the normalized power modulating functions can be explained by figuring out the effect of $q_{1}$ and $q_{2}$ in (12): as $q_{1}$ becomes bigger than $q_{2}$, the non-zero part of the modulating function goes to the left of the interval, and vice versa. The exponents $q_{1}$ and $q_{2}$ not only affect the position of the non-zero part of the modulating function but also its width, see Fig. 2. Thus, in our paper, we 


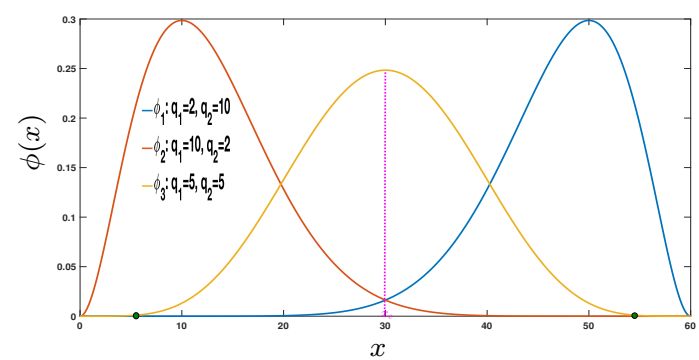

Fig. 2: Normalized power modulating functions with respect to different powers $q_{1}$ and $q_{2}$.

present how to construct well-localized modulating functions, and hence using the minimal set of modulating functions can be enough.

Remark 1. If $\phi(x)$ is a modulating function of order $l=\bar{n}$ and almost zero outside a finite interval, then $\phi^{(p)}(x)$ for $p=$ $0, \ldots, \bar{n}-1$, are almost zero outside the interval. However, since the matrix $\mathcal{A}$ in (7) involves $\phi^{(\bar{n})}(x)$ multiplied by the measurement, the previous observations suggest choosing $l \geq$ $\bar{n}+1$ to ensure that $\phi^{(\bar{n})}(x)$ is also almost zero outside the interval.

\section{B. Selection of Modulating Functions' Design Parameters}

It is worth to note that the center of the non-zero part of the normalized power modulating function given in (12) is mathematically given by:

$$
x_{c}=\underset{x}{\operatorname{argmax}} \phi(x)=\frac{L q_{2}}{q_{1}+q_{2}},
$$

see Fig. 2. We propose Algorithm IV.1 as a systematic way to construct a modulating function that has a non-zero part with a specified center $x_{c}$ and almost vanishes at a desired value $x_{\Delta}$ (e.g., at any of the small green points that are shown in the $x$-axis of Fig. 2) through tuning $q_{1}$ and $q_{2}$.

To examine the effectiveness of this algorithm, it is used to construct three modulating functions that have different centers, e.g., $x_{c_{1}}=20, x_{c_{2}}=30$ and $x_{c_{3}}=40$ (let $\left.X_{c}=\{20,30,40\}\right)$ and almost vanish at, e.g., $x_{\Delta_{1}}=5$, $x_{\Delta_{2}}=15$ and $x_{\Delta_{3}}=30$, respectively $\left(X_{\Delta}=\{5,15,30\}\right)$. Algorithm IV.1 gives the following values for $q_{1}$ and $q_{2}$ in the three modulating functions: $q_{1}=\{12,15,26.5\}$ and $q_{2}=\{6,15,53\}$; see Fig. 3 . The figure demonstrates the reliability of the algorithm.

\section{Proposed Selection Strategy}

Based on the previous observations on Fig. 1 (given in Subsection IV-A), only appropriate modulating functions should be used in the estimation. This will not only enhance the performance of the method, but also will give the possibility to reduce $M$ (i.e. reduce the size of the linear system), and hence reduce the computational load. We propose to use Algorithm IV.1 for constructing modulating functions based

\footnotetext{
${ }^{1}$ If a time-dependent modulating function is used, then $\bar{n}$ in the algorithm refers to the temporal derivative's order.
}
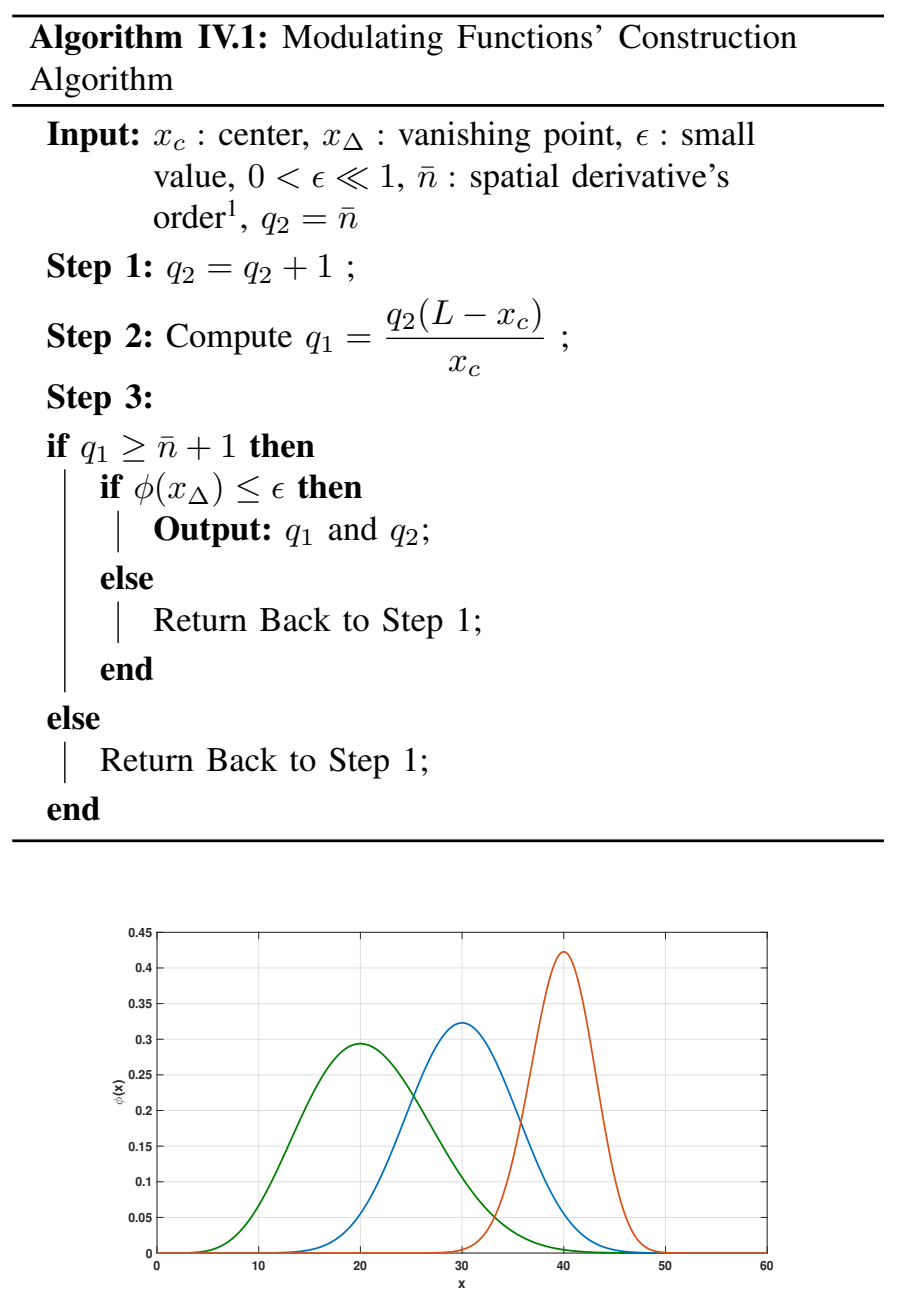

Fig. 3: Implementation of Algorithm IV.1 to construct modulating functions that have centers at $X_{c}=[20,30,40]$ and vanish at $X_{\Delta}=[5,15,30]$.

on the non-zero parts of the measurements. In other words, the non-zero parts of the modulating functions will be chosen to be localized around the informative parts (non zero part) of the measured signals. However, there is a large number of possible choices for the center $x_{c}$ and the vanishing point $x_{\Delta}$. Thus, we propose a strategy (Strategy 1) using the estimator variance given in [15] to select a set of well-localized modulating functions. First, we recall some notations and define new ones which are used in Strategy 1:

- $X_{c}$ is the set that contains different possible values for the centers $x_{c_{i}}$ of the modulating functions,

- $X_{\Delta}$ is the set that contains the corresponding suitable vanishing points $x_{\Delta_{i}}$,

- $I_{c}$ represents the size of $X_{c}$,

- $I_{u}$ represents the desired number of modulating functions such that $0 \leq I_{u} \leq I_{c}$ (Here we would like to have $I_{u}$ equals to the number of unknowns to obtain a square system)

- $I_{b}$ represents the number of $I_{u}$-combinations of the set $X_{c}$; hence, $I_{b}$ is equal to the binomial coefficient $\left(\begin{array}{l}I_{c} \\ I_{u}\end{array}\right)$,

- $X_{c}^{i}, i=1,2, \ldots, I_{b}$, represents the combination subsets 
TABLE I: Relative estimation errors for the coefficients $\alpha_{1}, \alpha_{2}$, and $\alpha_{3}$ in the fKdV equation when using the first $r$ modulating functions; $M=56$ and $q=13$.

\begin{tabular}{cccc}
\hline$r$ & $\frac{\left|\alpha_{1}-\hat{\alpha}_{1}\right|}{\left|\alpha_{1}\right|} \times 100$ & $\frac{\left|\alpha_{2}-\hat{\alpha}_{2}\right|}{\left|\alpha_{2}\right|} \times 100$ & $\frac{\left|\alpha_{3}-\hat{\alpha}_{3}\right|}{\left|\alpha_{3}\right|} \times 100$ \\
\hline 10 & 1028.7 & 51.299 & 56.081 \\
20 & 186.16 & 118.55 & 109.09 \\
30 & 73.516 & 69.418 & 166.12 \\
40 & 37.111 & 22.973 & 127.75 \\
45 & 2.0481 & 3.7241 & 29.395 \\
50 & 0.38058 & 2.576 & 2.8871 \\
56 & 0.53939 & 3.4406 & 8.6231 \\
\hline
\end{tabular}

of the set $X_{c}$,

- $X_{c}^{*}$ represents the combination subset that contains good values for the center $x_{c}$ (hence, good modulating functions).

\section{Strategy 1.}

1) Apply Algorithm IV.1 to construct $I_{c}$ modulating functions, $\left\{\phi_{m}\right\}_{m=1}^{I_{c}}$, based on the values in $X_{c}$ and $X_{\Delta}$.

2) Compute the estimator variance of the combination subsets $X_{c}^{i}, i=1,2, \ldots, I_{b}$.

3) Select the combination subset that gives the minimum variance:

$$
X_{c}^{*}=\underset{X_{c}^{i}, i=1, \ldots, I_{b}}{\operatorname{argmin}}[\operatorname{trace}(\operatorname{Var}[\tilde{\Gamma}])] .
$$
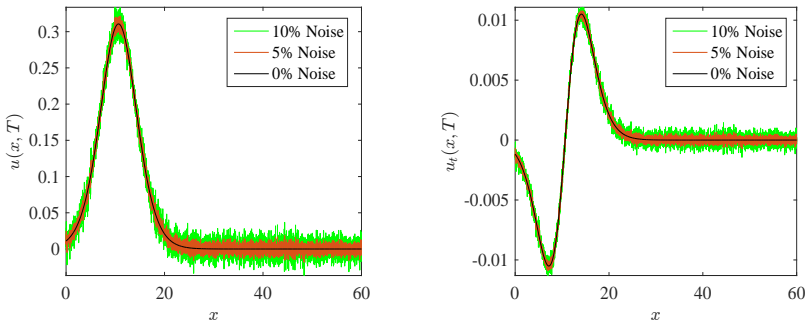

Fig. 4: Exact and noisy measurements of the fKdV equation with coefficients given in Example 1: black, brown, and green lines represent respectively the exact measurement, the ones with $5 \%$ of noise, and the ones with $10 \%$ of noise. $t^{*}=T$.

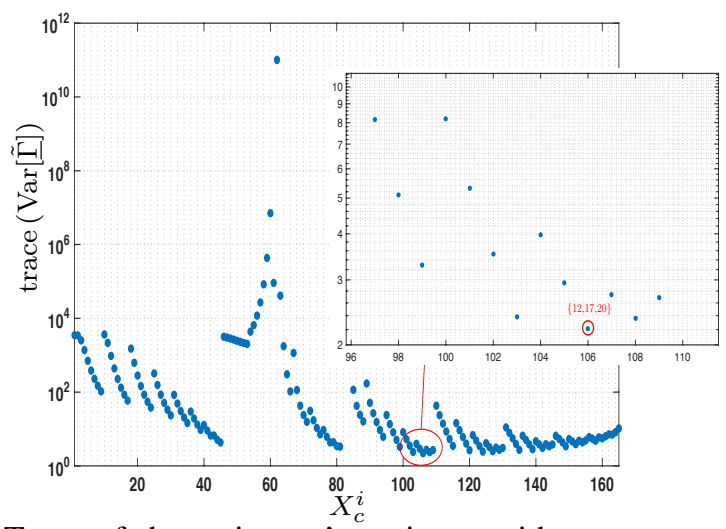

Fig. 5: Trace of the estimator's variance with respect to the different possible combinations $X_{c}^{i}, i=1,2, \ldots, I_{b}$, where $X_{c}=10,11, \ldots, 20, X_{\Delta}=X_{c}+7$, and noise level $=10 \%$.

and the vanishing points are valid: $X_{c}=10,11, \ldots, 20$ and $X_{\Delta}=X_{c}+7$. Using Strategy 1, the combination that gives the minimal variance is at $X_{c}^{*}=\{12,17,20\}$, see Fig. 5. The estimation errors at these constructed and selected modulating functions are given in Table II. The results are good and even better than the ones obtained in [15] when $M$ was selected based on the variance (compared with Table 1 in [15]).

2) Example 2: $\alpha_{1}=\frac{105}{16}, \alpha_{2}=\frac{13}{4}, \alpha_{3}=1$

Based on the measurements of this example which are shown in Fig. 6, the following values for the centers and the vanishing points are chosen: $X_{c}=12,13, \ldots, 28$ and $X_{\Delta}=X_{c}+5$. Strategy 1 gives $X_{c}^{*}=\{16,17,21\}$. The relative errors of the estimation are given in Table III.

3) Example 3: $\alpha_{1}=5, \alpha_{2}=3, \alpha_{3}=4$

The measurements are depicted in Fig. 7. From the figure, we

TABLE II: Relative estimation errors in Example 1 using Strategy 1.

\begin{tabular}{lccc}
\hline $\begin{array}{l}\text { Noise } \\
(\%)\end{array}$ & $\frac{\left|\alpha_{1}-\hat{\alpha}_{1}\right|}{\left|\alpha_{1}\right|} \times 100$ & $\frac{\left|\alpha_{2}-\hat{\alpha}_{2}\right|}{\left|\alpha_{2}\right|} \times 100$ & $\frac{\left|\alpha_{3}-\hat{\alpha}_{3}\right|}{\left|\alpha_{3}\right|} \times 100$ \\
\hline 0 & $7.7716 \mathrm{e}-13$ & $4.1078 \mathrm{e}-13$ & $1.3212 \mathrm{e}-12$ \\
1 & 0.0039529 & $2.1162 \mathrm{e}-05$ & 0.36086 \\
3 & 0.011386 & 0.0013892 & 1.0678 \\
5 & 0.018171 & 0.004777 & 1.7553 \\
10 & 0.032154 & 0.022213 & 3.39 \\
\hline
\end{tabular}



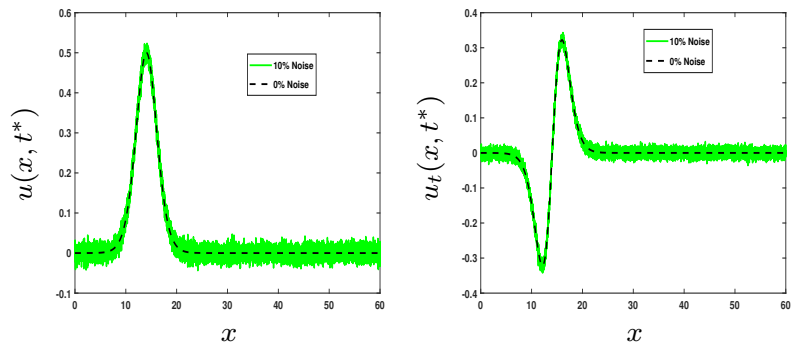

Fig. 6: Exact and noisy measurements of the $\mathrm{fKdV}$ equation with coefficients given in Example 2, $t^{*}=\left\lfloor N_{t} / 8\right\rfloor$.

TABLE III: Relative estimation errors in Example 2 using $X_{c}^{*}=\{16,17,21\}$ (given by Strategy 1 ).

\begin{tabular}{lccc}
\hline $\begin{array}{l}\text { Noise } \\
(\%)\end{array}$ & $\frac{\left|\alpha_{1}-\hat{\alpha}_{1}\right|}{\left|\alpha_{1}\right|} \times 100$ & $\frac{\left|\alpha_{2}-\hat{\alpha}_{2}\right|}{\left|\alpha_{2}\right|} \times 100$ & $\frac{\left|\alpha_{3}-\hat{\alpha}_{3}\right|}{\left|\alpha_{3}\right|} \times 100$ \\
\hline 0 & $2.1384 \mathrm{e}-12$ & $3.5814 \mathrm{e}-11$ & $1.9873 \mathrm{e}-10$ \\
1 & 0.091894 & 0.29428 & 0.17276 \\
3 & 0.27341 & 0.87403 & 0.50608 \\
5 & 0.45192 & 1.4421 & 0.82337 \\
10 & 0.88523 & 2.812 & 1.5482 \\
\hline
\end{tabular}

can set, $X_{c}=15,16, \ldots, 35$ and $X_{\Delta}=X_{c}+8$. Strategy 1 candidates $X_{c}^{*}=\{22,31,35\}$. The relative errors of the estimation are given in Table IV.

The results in the previous examples demonstrate the effectiveness and efficiency of Strategy 1 in selecting good modulating functions, and hence in improving the estimation results with less computational cost.

\section{CONCLUSION}

In this paper, an off-line algorithm has been proposed to construct modulating functions based on the measurements supports. Then, based on the estimator variance, a set of
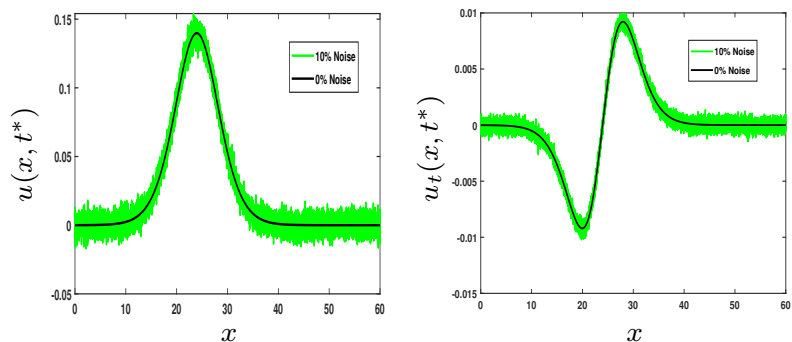

Fig. 7: Exact and noisy measurements of the $\mathrm{fKdV}$ equation with coefficients given in Example $3, t^{*}=T$.

TABLE IV: Relative error estimation in Example 3 using $X_{c}^{*}=\{22,31,35\}$ (given by Strategy 1 ).

\begin{tabular}{lccc}
\hline $\begin{array}{l}\text { Noise } \\
(\%)\end{array}$ & $\frac{\left|\alpha_{1}-\hat{\alpha}_{1}\right|}{\left|\alpha_{1}\right|} \times 100$ & $\frac{\left|\alpha_{2}-\hat{\alpha}_{2}\right|}{\left|\alpha_{2}\right|} \times 100$ & $\frac{\left|\alpha_{3}-\hat{\alpha}_{3}\right|}{\left|\alpha_{3}\right|} \times 100$ \\
\hline 0 & $2.7534 \mathrm{e}-12$ & $6.8123 \mathrm{e}-11$ & $1.7801 \mathrm{e}-10$ \\
1 & 0.020309 & 0.09684 & 0.075829 \\
3 & 0.061615 & 0.29076 & 0.22022 \\
5 & 0.10384 & 0.48502 & 0.35497 \\
10 & 0.21343 & 0.9721 & 0.64999 \\
\hline
\end{tabular}

modulating functions is chosen. The number of the modulating functions in the set is equal to the number of unknowns, which simplifies the problem into solving a square algebraic system. The good performance of the proposed strategy has been demonstrated numerically. In a future work, the proposed algorithm will be extended for real-time estimation.

\section{REFERENCES}

[1] S. Asiri, S. Elmetennani, and T.-M. Laleg-Kirati, "Moving-horizon modulating functions-based algorithm for online source estimation in a first-order hyperbolic partial differential equation," Journal of Solar Energy Engineering, vol. 139, no. 6, p. 061007, 2017.

[2] A. Ionesi and J. Jouffroy, "On-line parameter estimation of reducedorder models for buildings energy dynamics using the modulating function method," in 2018 Winter Simulation Conference (WSC). IEEE, 2018, pp. 503-514.

[3] Y.-Q. Wei, D.-Y. Liu, and D. Boutat, "Innovative fractional derivative estimation of the pseudo-state for a class of fractional order linear systems," Automatica, vol. 99, pp. 157-166, 2019.

[4] D.-Y. Liu, T.-M. Laleg-Kirati, W. Perruquetti, and O. Gibaru, "Nonasymptotic state estimation for a class of linear time-varying systems with unknown inputs," in the 19th World Congress of the International Federation of Automatic Control. IEEE, 2014, pp. 3732-3738.

[5] J. Jouffroy and J. Reger, "Finite-time simultaneous parameter and state estimation using modulating functions," in 2015 IEEE Conference on Control Applications (CCA). IEEE, 2015, pp. 394-399.

[6] X. Wei, D.-Y. Liu, and D. Boutat, "Nonasymptotic pseudo-state estimation for a class of fractional order linear systems," IEEE Transactions on Automatic Control, vol. 62, no. 3, pp. 1150-1164, 2016.

[7] M. Mboup, C. Join, and M. Fliess, "Numerical differentiation with annihilators in noisy environment," Numerical algorithms, vol. 50, no. 4, pp. 439-467, 2009.

[8] D.-y. Liu, O. Gibaru, and W. Perruquetti, "Parameters estimation of a noisy sinusoidal signal with time-varying amplitude," in the 19th Mediterranean Conference on Control \& Automation. IEEE, 2011, pp. 570-575.

[9] I. N'Doye, S. Asiri, A. Aloufi, A. Al-Awan, and T.-M. Laleg-Kirati, "Intelligent proportional-integral-derivative control-based modulating functions for laser beam pointing and stabilization," IEEE Transactions on Control Systems Technology, vol. 28, no. 3, 2020.

[10] H. Preisig and D. Rippin, "Theory and application of the modulating function methodi. review and theory of the method and theory of the spline-type modulating functions," Computers \& Chemical Engineering, vol. 17 , no. 1 , pp. $1-16,1993$.

[11] S. Asiri and T.-M. Laleg-Kirati, "Modulating functions-based method for parameters and source estimation in one-dimensional partial differential equations," Inverse Problems in Science and Engineering, vol. 25, no. 8, pp. 1191-1215, 2017.

[12] D.-Y. Liu and T.-M. Laleg-Kirati, "Robust fractional order differentiators using generalized modulating functions method," Signal Processing, vol. 107, pp. 395-406, 2015.

[13] G. Pin, B. Chen, and T. Parisini, "Robust finite-time estimation of biased sinusoidal signals: A volterra operators approach," Automatica, vol. 77, pp. 120-132, 2017.

[14] Y. Tian, Y.-Q. Wei, D.-Y. Liu, and D. Boutat, "Fast and robust estimation for positions and velocities from noisy accelerations using generalized modulating functions method," Mechanical Systems and Signal Processing, vol. 133, p. 106270, 2019.

[15] S. Asiri, D.-Y. Liu, and T.-M. Laleg-Kirati, "Modulating functions method for parameters estimation in the fifth order kdv equation," in the 25th Mediterranean Conference on Control and Automation. IEEE, 2017, pp. 30-35.

[16] M. Shinbrot, "On the analysis of linear and nonlinear systems," Trans. ASME, vol. 79, no. 3, pp. 547-552, 1957.

[17] M. Noack, J. G. Rueda-Escobedo, J. Reger, and J. A. Moreno, "Fixedtime parameter estimation in polynomial systems through modulating functions," in 2016 IEEE 55th Conference on Decision and Control (CDC). IEEE, 2016, pp. 2067-2072.

[18] Y. Yamamoto and É. I. Takizawa, "On a solution on non-linear timeevolution equation of fifth order," Journal of the Physical Society of Japan, vol. 50, no. 5, pp. 1421-1422, 1981. 